\title{
Fabrication of bioinspired edible liquid marble with phase transition and tunable water barrier property
}

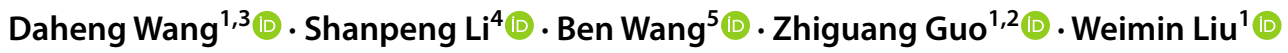

Received: 4 March 2021 / Accepted: 4 July 2021 / Published online: 4 August 2021

(c) Zhejiang University Press 2021

\begin{abstract}
Based on aphid wax-honeydew marble and the hydrophobic wax structure of lotus and its derived applications with superareophilic and superhydrophobic properties, edible carnauba wax and beeswax particles were mixed and utilized to mimic lotus wax and wrap liquid, thus forming liquid marbles (LMs). Through the utilization of continuous production system (CPS), wax as an interfacial surfactant, water and solid, air-phase or mixed-phase marble content was produced. The edible liquid marble (ELM) could encapsulate water and food droplets. Edible solid marble (ESM) and edible solid hollow marbles (ESHMs) could be fabricated by applying pectin or syrup. Moreover, through the heating of wax powders with different melting temperatures, stable tablets and hollow capsules could be produced. The wax powder as interfacial surfactant could firmly bind with pectin through hydrogen bonds on ESM. The edible LMs can therefore be applied for residue reduction, corrosion reduction, biohazard prevention and cleaning in the food industry. The other phase LMs could act as novel tools in the pharmaceutical and food industries with the above-mentioned water transport, preservation, sustained releasing and selective releasing abilities.
\end{abstract}

\section{Graphic abstract}

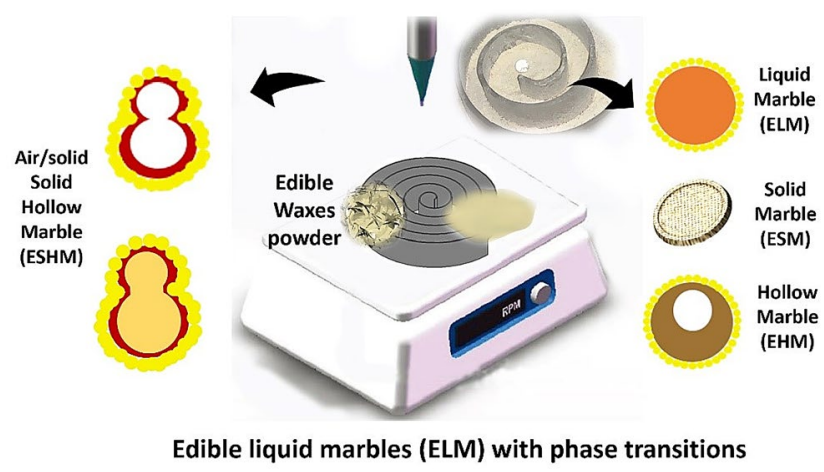

Keywords Wax particle mixture $\cdot$ Edible $\cdot$ Hydrophobic powder $\cdot$ Liquid marble $\cdot$ Phase transition $\cdot$ Food

Zhiguang Guo

zguo@licp.cas.cn

1 State Key Laboratory of Solid Lubrication, Lanzhou Institute of Chemical Physics, Chinese Academy of Sciences, Lanzhou 730000, China

2 Ministry of Education Key Laboratory for the Green Preparation and Application of Functional Materials, Hubei University, Wuhan 430062, China
3 University of Chinese Academy of Sciences, Beijing 100039, China

4 College of Engineering, Lishui University, Lishui 323000, China

5 College of Chemistry and Environmental Engineering, Shenzhen University, Shenzhen 518060, China 


\section{Introduction}

Inspired by aphid wax-honeydew marble [1, 2], researches have been conducted on liquid marbles (LM) formed through wrapping water droplets with hydrophobic particles. LM have been applied in various fields such as gas sensing [3, 4], adhesive materials [5], microbioreactors [6] and water pollution indicators/collectors $[7,8]$. The stability of LM can be related to particle size distribution, surface energy and external conditions [9-11]. Moreover, the LM could be further processed through gravity [12], mechanical force $[13,14]$, magnetic manipulation [8, 15-17] and light-driven locomotion [18, 19]. However, the low-surface-energy materials and surfactant in the air-water interface used for the LMs to encapsulate liquid have been mainly perfluorinated compounds (PFCs) [20]. These harmful materials could be replaced by hydrophobic carnauba wax and beeswax particles that mimic lotus wax [21-23], which are also edible (generally recognized as safe (GRAS) under regulation 21CFR184.1973 and 1978). Biohazard and public health issues have triggered a long-standing debate with regard to human daily life [24]. Recent events of COVID-19 transmission through food produce and supply chains at food markets can also result from infected manpower and unprotected/unsensitized production [25-27]. Therefore, it is essential for novel methods and automated production to replace human labour in food production [28]. Edible liquid marble (ELM) and its derived applications could greatly reduce the need for manpower through automated and direct wrapping of food liquid content and other areas, such as tablet and capsule production in the pharmaceutical industry to eliminate bacterial growth, viral contamination or material residue. Therefore, the application of ELM could be extended to other fields such as additive food packaging, candy making, beverage production, pharmaceutical drugs and food supplements. LM and its derived phased marbles could be an interesting research direction in this context.

Edible packaging materials have attracted great attention in recent years, whereas edible LM remains to be explored [29]. Kawamura et al. introduced ELM with fatty acid crystals and triacylglycerol crystals, and indicated its use for food or healthcare products. However, practical experiments were lacking from their study, and wax as a temperature-sensitive hydrophobic material remained to be investigated in the field of ELM [30]. Therefore, in the present work, the procedure of wax powder fabrication is proposed through separating and drying wax from wax-in-ethanol emulsion. Carnauba wax and beeswax with different melting temperatures $\left(82{ }^{\circ} \mathrm{C}\right.$ and $62-64{ }^{\circ} \mathrm{C}$, respectively) were mixed and extracted in powder form and wrapped in liquid to form ELMs. These two types of wax have been described and applied as a superhydrophobic coating for several applications: food liquid residue reduction [31, 32], food packaging materials [33] or direct food coating [34]. Studies have focused on enhancing the stability or practicality of the wax made into superhydrophobic materials by adding an elastic layer in between coating and substrate [35], controlling the melting of wax coating [33, 36] or fumigating wax onto substrate [37]. However, most of these researches relied on spraying or fumigation that could uniformly distribute the coating. The downside of these techniques is that they can only be applied to surfaces that are flat and adhesive with regard to wax such as metal, glass and mostly plastics [31, 32, $35,37]$. On the other hand, independent hydrophobic wax powder could serve as a superhydrophobic and superareophilic surface surfactant between the water-air interface of LM and could facilitate the application of edible liquid marble (ELM) in the food industry and pharmaceutical fields. The single-wax-in-ethanol emulsion has been extensively studied for producing bioinspired superhydrophobic coatings; however, the properties and application of mixed superhydrophobic wax powder and its derivatives need to be explored, and the heating effect of mixed wax remains to be researched.

The mass production of LM is essential for practical application, which can be achieved by the simplification and modification of the previously studied coalescence and splitting system (CSS) and the design of a continuous production system (CPS) [12]. Furthermore, taking advantage of the enlarged spiral wall of CPS and applying edible pectin (GRAS, 21CFR184.1588) as a LM component, a series of other phased marbles can be fabricated, as the pectin solution is highly viscous and commonly serves as adsorbent, bulk-forming agent and excipient [38-40]. Thus, in this article, edible solid marble (ESM) was be fabricated through the simple drying of pectin ELM. Moreover, through simple air injection at the enlarged spiral wall of CPS and subsequent drying, mixed-phase edible hollow marble (EHM) and edible solid hollow marble (ESHM) were produced. The ESHM could further encapsulate oil or solid through a drilled hole that was repaired. In this manner, the CPS, ESM, EHM and ESHM could further expand the function and application of traditional LM.

Aimed at further characterizing the practicality of the heated and unheated ESM and ESHM, in vitro digestion tests were conducted. An in-vial shaking test was also conducted to evaluate the durability of ESM and ESHM in transport and storage situations. The ESM and ESHM can be applied in both food and pharmaceutical industry applications for human consumption and further fields. The application of ESM and ESHM can endow the industry with progress in simplifying automated production and the transportation of water, novel food, drugs or supplements, 
as well as the reduction of biohazard and waste in pipeline and industry transportation processes.

\section{Experimental procedure}

\section{Fabrication of edible hydrophobic mixed wax powder}

The wax-in-ethanol solutions of carnauba wax (purchased from Shanghai Macklin Biochemical Co. Ltd., China) and beeswax (obtained from Solarbio Science \& Technology Co. Ltd., China) were prepared using methods previously reported by our research group but with modifications $[33,41]$. Specifically, $2 \mathrm{~g}$ of carnauba wax and beeswax was separately added into flasks with $200 \mathrm{~mL}$ of ethanol (purchased from Rionlon, China). Next, wax-in-ethanol solutions of carnauba wax and beeswax were heated to dissolve the wax. The solutions were cooled at room temperature for the precipitation of edible hydrophobic wax particles. Subsequently, the wax particle suspensions were ultrasonicated for $2 \mathrm{~h}$ for the even separation and distribution of particles.

The two obtained solutions were mixed with carnauba wax in a beeswax ratio of $4: 1,3: 1$ and $2: 1$. The wax-inethanol solutions with different mixture ratios were kept in an oven at $30^{\circ} \mathrm{C}$ to evaporate the ethanol. Deionized water (purified by the ModuPure system) was added to the dried wax mixtures to prepare wax-water suspensions. The dried edible hydrophobic mixed wax powders with different wax ratios were collected after drying at $30{ }^{\circ} \mathrm{C}$. Carnauba wax powder can be also prepared using this drying method.

\section{Fabrication of edible liquid marbles and their phase transition (liquid, solid and hollow marbles)}

The dried edible hydrophobic mixed wax powder was evenly distributed onto the magnified spiral wall dish described in our recent work [12]. Next, edible liquid marbles (ELMs) were fabricated through pipetting liquid of a specified volume on the centre of the spiral dish and agitation on an orbital shaker. ELMs can pack liquid such as water, orange syrup, ketchup and pectin. The pectin solution was prepared through dissolving pectin powder (purchased from Shanghai Macklin Biochemical Co. Ltd., China) in distilled and deionized water at room temperature to form a $10 \% \mathrm{w} / \mathrm{v}$ solution. ESM can be fabricated through the drying of orange syrup or pectin ELMs and forming candies or tablets. Edible solid hollow marble (ESHM) can be further produced through the air injection and drying of pectin ELM.

\section{Bacteria detection using a luminometer}

A 3 M Clean-Trace NG Luminometer was applied as the relative light unit of bacterial fluorescence and the indication of bacterial growth. A $133 \mathrm{~mm} \times 24.5 \mathrm{~mm}$ area of both the direct and the ELM transport pipeline was scrubbed with $3 \mathrm{M}^{\mathrm{TM}}$ Clean-Trace ${ }^{\mathrm{TM}}$ Surface Swab.

\section{In vitro digestion experiment}

This experiment was designed according to the method proposed by Gawlik-Dziki et al. [42]. The ESM and EHM were subjected to 3 stages of in vitro digestion. Stage I mimicked oval digestion: shaking in simulated saliva addition $(30 \mathrm{~mL})$ for $10 \mathrm{~min}$ in $37^{\circ} \mathrm{C}$; Stage II mimicked stomach digestion: addition of simulated gastric fluid (Coolaber Co., Ltd., Beijing, China), $\mathrm{pH}$ change to 1.2 by adding $5 \mathrm{M} \mathrm{HCl}$ and shaking for $120 \mathrm{~min}$ at $37{ }^{\circ} \mathrm{C}$; and Stage III mimicked intestine digestion: $\mathrm{pH}$ change to 6 by $0.1 \mathrm{M}$ of $\mathrm{NaHCO}_{3}$, addition of bile extract and pancreatin mixture, $\mathrm{pH}$ adjustment to 7 with $1 \mathrm{M} \mathrm{NaOH}, 5 \mathrm{~mL}$ of $120 \mathrm{mM} \mathrm{NaCl}$ addition, $5 \mathrm{~mL}$ of $5 \mathrm{mM}$ $\mathrm{KCl}$ addition and shaking for $60 \mathrm{~min}$ at $37^{\circ} \mathrm{C}$. All samples were shaken by an electrothermal oscillating sink (DKZ-2 Jinghong Co., Ltd., China).

\section{Mechanical stability test}

Pectin-made ESM, ESHM and heated ESM, ESHM were selected for an in-vial stability test. Approximately 20 of the selected subjects were encased into small vials with an additional $1 \mathrm{~g}$ of wax powder for protection. Shaking by electrothermal oscillating sink (DKZ-2 Jinghong Co., Ltd., China) was then performed for $2 \mathrm{~h}$. The samples in 4 vials were selected for water floating test and the determination of coating integrity.

\section{Characterization}

The image acquisition of Au-sputtered ESM, EHM and cross-sectional specimens was performed by field emission scanning electron microscope (FESEM, QUANTA FEG 650, FEI, USA). Images of wax particles were captured through transmission electron microscopy (TEM, FEI Tecnai G2 F30). The FTIR spectra results were collected using Fourier transform infrared spectroscopy (FTIR, Thermo Scientific Nicolet iS10). The mechanical testing of ESM and EHM was conducted through in-vial shaking. The stability of the mixed wax coating was characterized by its floating ability. Magnified ELM, ESM and EHM images were measured by the JC2000D system (Zhongchen Digital Equipment Co., Ltd., Shanghai, China). Pipette injectors of $1-5 \mu \mathrm{L}$ and $10-100 \mu \mathrm{L}$ volume (Dragon Laboratory Instruments Co., Ltd., Beijing, China) were applied for all liquids. 
The water contact angle (WCA) of $5 \mu \mathrm{L}$ water droplet and magnified images were measured using the JC2000D system (Zhongchen Digital Equipment Co., Ltd., Shanghai, China). The WCA recordings of each sample were recorded at five different positions and averaged for result.

\section{Results and discussion}

\section{Fabrication of edible wax powder}

The edible hydrophobic powder was prepared through two stage evaporations of wax-in-ethanol emulsion. As the previously conducted experiment indicated that both the sprayed coatings of emulsion-prepared carnauba wax and beeswax particles could possess superhydrophobicity [33, 41], when water droplet is dropped onto the powder with superhydrophobicity and superareophilicity, the particles act as a surfactant at the water-air interface and have the tendency to warp the droplet for surface free energy minimization to form liquid marble (LM). The SEM images in Figs. 1a and $1 \mathrm{~b}$ demonstrate that the as-prepared particles possess microscale diameter and nanoscale porous structure; therefore, they ascribe water repellent ability to the powder mixture [43]. Furthermore, the FTIR result of both waxes indicates abundant stretching vibrations of $-\mathrm{CH}_{3},-\mathrm{CH}_{2}$ groups at wave numbers 2920 and $2850 \mathrm{~cm}^{-1}$ and an ester group stretching vibration at $1750 \mathrm{~cm}^{-1}$. The corresponding ester group and aliphatic groups provide the particles with hydrophobic chemical property (Fig. S1) [44].

When the result is combined with previous research, the diameter of the carnauba wax and beeswax can be concluded as: $d_{\mathrm{c}}=5.6 \pm 1.2 \mu \mathrm{m}$ and $d_{\mathrm{b}}=6.2 \pm 1.5 \mu \mathrm{m}$ (Fig. S2). Models of the wax are presented in Fig. 1c. As shown in Fig. 1d, e and Table S1, the water contact angle (CA) on heated wax powder with $3: 1$ mixing ratio reaches $151^{\circ}$; thus, it is deemed suitable for LM production. Due to the stability of chemical properties of wax, wax powder could also wrap liquid with different $\mathrm{pH}$ values between 1 and 14 (Fig. 1f). As shown in Fig. 1g, LMs with different $\mathrm{pH}$ values were formed into "LM" symbols on hydrophilic paper surface, indicating that the wax powder is suitable for liquid foods with various $\mathrm{pH}$ values.

As established previously, the heating of the beeswax to $55{ }^{\circ} \mathrm{C}$ could melt the wax without chemical changes and strengthen the wax coating integrity through dense coating formation (Fig. 2). The heating time, however, requires precise control to maintain the superhydrophobicity of the coating [33]. Therefore, to further simplify the heating process and perform the heating process together with wax powder production, carnauba wax with higher melting point was introduced as a superhydrophobic convex structure [41]. Mixing ratios were set separately to $4: 1,3: 1$ and 2:1. These ratios were set, because a ratio of $1: 1$ or higher may not lead to powder formation, and a ratio of 4:1 or higher may not yield a protective beeswax layer after heating (Table S1).
Fig. 1 Properties of wax particles and wax powders. a SEM image of carnauba wax. b SEM image of beeswax. $\mathbf{c}$ Models illustrating the sizes and morphologies of carnauba wax and beeswax particles. d SEM image showing the surface morphology of carnauba wax and beeswax mixture (ratio $3: 1$ ). e Optical images of the heated mixed wax particles (ratio 3:1) and water contact angle on the heated mixed wax surface at room temperature. $\mathbf{f}$ Optical image of $40 \mu \mathrm{L}$ LMs with $\mathrm{pH}=1$ and $\mathrm{pH}=14$ coloured solutions. g Optical image of fourteen ELMs with $40 \mu \mathrm{L}$ solutions of different $\mathrm{pH}$ values ranging from 1 to 14 , forming "LM" characters (a)

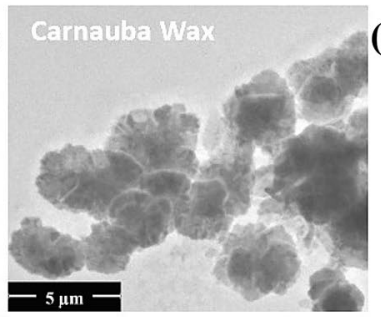

(d)

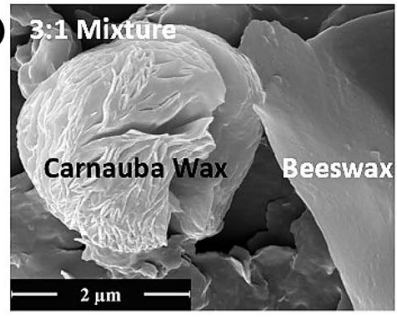

(f)

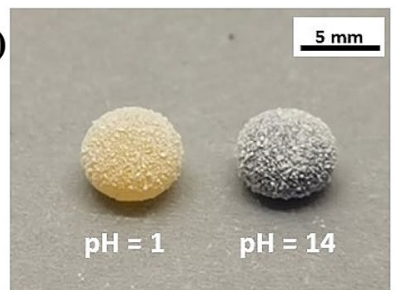

(b)
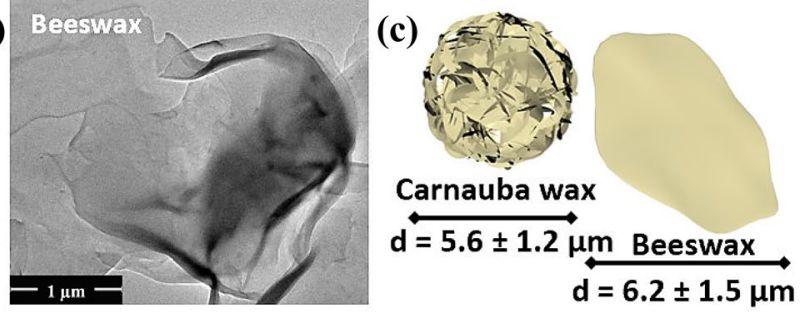

(e)

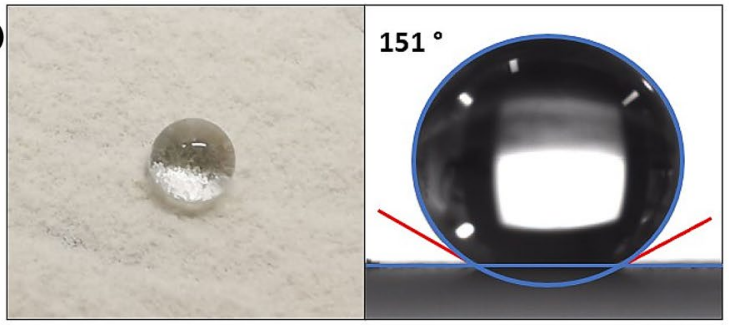

(g)

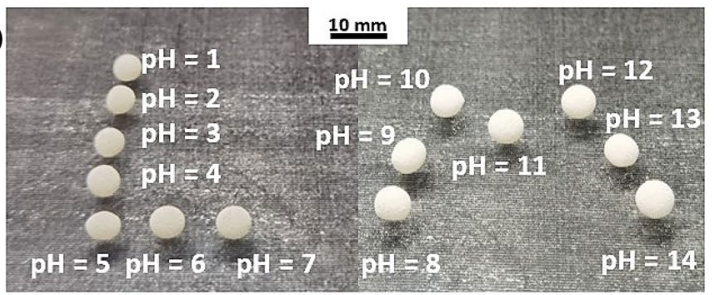



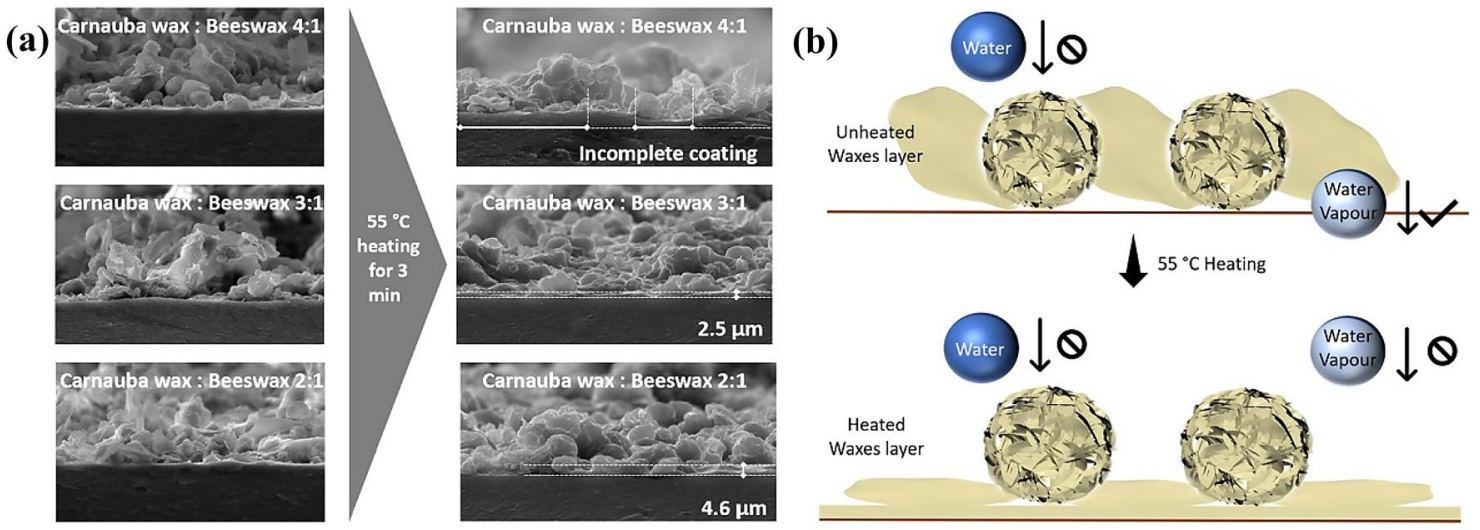

Fig. 2 Illustration of heating effect on wax coating. a SEM images of coatings of wax with different mixing ratios on the pectin surface in both the unheated and heated $\left(55^{\circ} \mathrm{C}\right)$ stage. b Schematic diagram of selected heating procedure of beeswax in the form of mixed wax particle powder

The beeswax layer may act as oxygen and vapour barrier preventing the disintegration of pectin ESM and ESHM. The mixed wax powder could allow the tablet or capsule of drug prepared in the form of ESM and ESHM to have the designated releasing capacity.

The SEM results indicated that the ratios of 2:1 and 3:1 could lead to uniform and dense coating with thickness of 4.6 and $2.5 \mu \mathrm{m}$, respectively, while the ratio of $4: 1$ or higher ratio could not (Fig. 2a). Therefore, as illustrated in Fig. S3, the heated 4:1 ESM deformed and eventually dissolved similarly to the unheated 4:1 ESM. While the heated 3:1 ESM remained on the water due to dense wax coating, only the powder with 3:1 wax ratio could possess superhydrophobicity, while the one with 2:1 ratio lost its superhydrophobicity through beeswax melting (Fig. S4). Therefore, the mixture with 3:1 ratio was selected for wax powder production.

\section{Applications of edible liquid marbles and their transition phases}

The continuous production system (CPS) was modified according to the coalescence and splitting system (CSS) described in previous work [12]. The CPS reduced the coalescence and splitting function and featured an enlarged spiral wall. The coalescence and splitting function was designed for a miniature bioreactor; therefore, the function of CPS was mainly focused at the mass production of liquids, especially edible food liquids. As illustrated in Fig. 3, CPS includes an orbital shaker, ELM collection plate, enlarged spiral wall and pipette sampler of the droplet. The edible wax powder was evenly distributed around the spiral, especially at the central area. The CPS could form ELM from 5 to $100 \mu \mathrm{L}$ water droplets using a pipette. At the beginning of ELM fabrication, CPS operates at a lower rotating speed of $100 \mathrm{r} / \mathrm{min}$. After the rough coating of the droplet, the CPS accelerates to $250 \mathrm{r} / \mathrm{min}$ and steers the ELM around the spiral wall for further powder coating and ELM distribution. The ELM was formed through dropping of the liquid onto the centre of the spiral wall followed by centrifugal forcedriven circular motion with gradually increased radius, thus allowing the coating, transportation and collection of the ELM with a production rate of about $1.5 \pm 0.4 \mathrm{~mL} \cdot \mathrm{min}^{-1}$. From the optical microscopic images of ELM with different focus layers, shown in Fig. S2, it is seen that the liquid can be firmly coated by granules of carnauba wax and beeswax mixture with sizes around $15.1 \pm 13.5 \mu \mathrm{m}$. Compared with the traditional manual dish rotating method, the CPS can uniformly coat the liquid marble through mass production with automatic operation [45]. Figure S3 presents the optical images of water, ketchup, orange syrup and pectin ELMs, where the macroscopic diameter of ELMs with different volumes are selectively shown. As seen in the figure, the wax powder was evenly distributed and conferred the ELM round shape for minimized surface energy. Since the thickness of wax coating has considerable impact on the liquid marble at small liquid volumes, the wax percentage significantly dropped with the increase in the liquid volume. The CPS system could efficiently mass produce and distribute ELMs with different droplet content and produce EHMs after air injection.

The ELMs and ESHM produced by CPS could be applied in the food industry and pharmaceutical industry according to applications 1-3 (Fig. 3). Application 1 preliminarily realizes the wrapping of food liquid such as ketchup and orange syrup. The autoformation of candy fabrication via dropping raw material onto CPS and directly producing manipulatable orange syrup ELM could also be accomplished, thus reducing biohazard during manual production. Application 2 utilizes the pectin as a plasticizer and the hydrogen binding force between wax powder and pectin to fabricate the pectin ESM. Furthermore, pectin is a natural drug-compatible plasticizer and sustained releasing agent $[46,47]$; therefore, 
Fig. 3 Illustration of the continuous production system (CPS) and its function

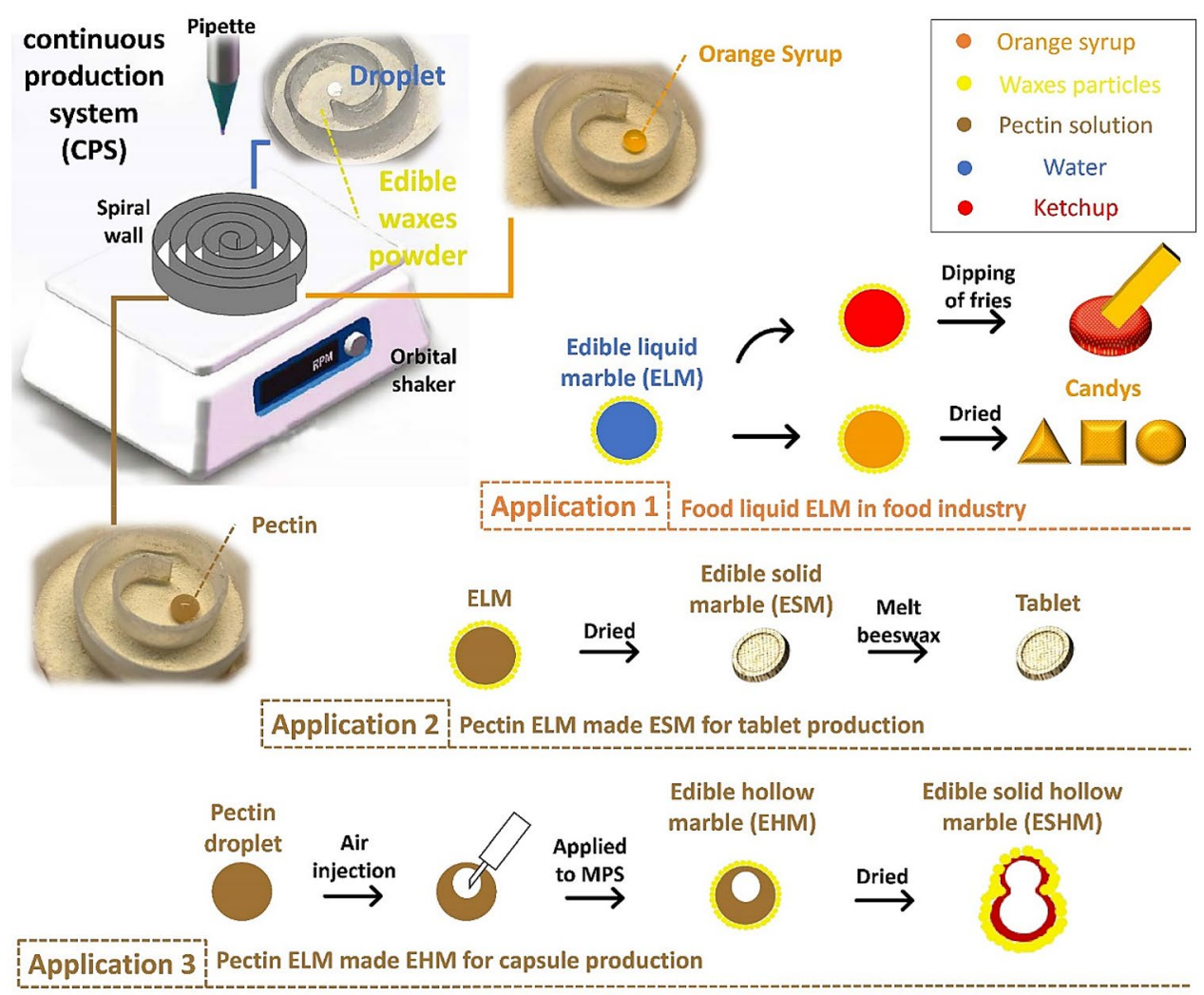

pectin ESM can serve as a drug in tablet form for pectincompatible and water-soluble medicine. Application 3 further utilizes the high viscosity property of the pectin solution (Table S2) $\left(\eta_{\text {pectin }}=6679 \mathrm{MPa} \cdot \mathrm{s}\right.$, at $25^{\circ} \mathrm{C}, \eta$ as dynamic viscosity). The EHM and ESHM can be fabricated by injecting air into pectin ELM. The ESHM possesses a hollow chamber, which enables the utility of ESHM such as a capsule for water-insoluble medicine or a supplement including fish oil.

\section{Packing of food liquids as edible liquid marbles (ELMs)}

ELM, a novel approach for packing food liquids, may serve as an innovative material in the field of food processing, production, transportation and consumption. The top view images of the magnified spiral wall and droplet of water, ketchup and orange syrup are shown in Fig. 4a. The ELM volume was precisely controlled at $100 \mu \mathrm{L}$, and the wax particle was evenly coated around the ELM. Moreover, as illustrated in Figs. 4b and S7, the diameter and height of ELM increase with the liquid volume (water, ketchup, orange syrup and pectin). A similar pattern can be found for the superhydrophobic surfaces; however, with the varying density of food liquid, the content of thicker liquid results in higher diameter-to-height ratios [48]. In this study, however, the orange syrup ELM retained its free rolling ability, indicating that when the volume ranged under $100 \mu \mathrm{L}$, the viscosity had an insignificant effect on ELM stability.
As a novel food additive packing method, the ketchup ELM can replace conventional plastic packaging, thereby reducing residual waste. As shown in Fig. 4c, the wax powder was evenly coated around $100 \mu \mathrm{L}$ of ketchup ELM allowing its subsequent transportation and storage. For the scenario of consumption, the ketchup was selected for flavouring fries. The dipping process of fries was recreated as shown in Fig. 4c. In the figure, the pancake shape represents the maximum disintegration point indicating the maximum increase in the surface area, thus causing shortage of superhydrophobic powder cover, defects and bare liquid regions [49]. For the reason mentioned above, when the ketchup ELM was pressed and reached the breaking point of pancake shape, the successful dipping of fries was achieved (Movie $\mathrm{S} 1)$. On the other hand, in the novel ELM candy production procedure, the orange syrup ELM could be directly placed onto moulds from CPS production and produce candies after heating (Fig. 4d). As indicated in Fig. 2b, the wax powder formed a dense beeswax layer to prevent the penetration of water and water vapour prolonging the shelf life of heated candies. Since beeswax and carnauba wax have been traditionally applied as glazing agents of candies or rough coating of crops $[50,51]$, any flavour changes of the ELM candies could be disregarded. The further exploration of ELM food applications presented in Figs. S8 and S9 indicates the potential prospects of wax powder ELMs. Orange syrup ELM could directly participate in food production 
Fig. 4 Liquid marble characterization and applications. a Images of the ketchup and orange syrup ELMs formation process. b Diameter-heightliquid volume relation of water ELM. c Dipping process of ketchup ELM. d Illustration and images of orange flavoured candy production process
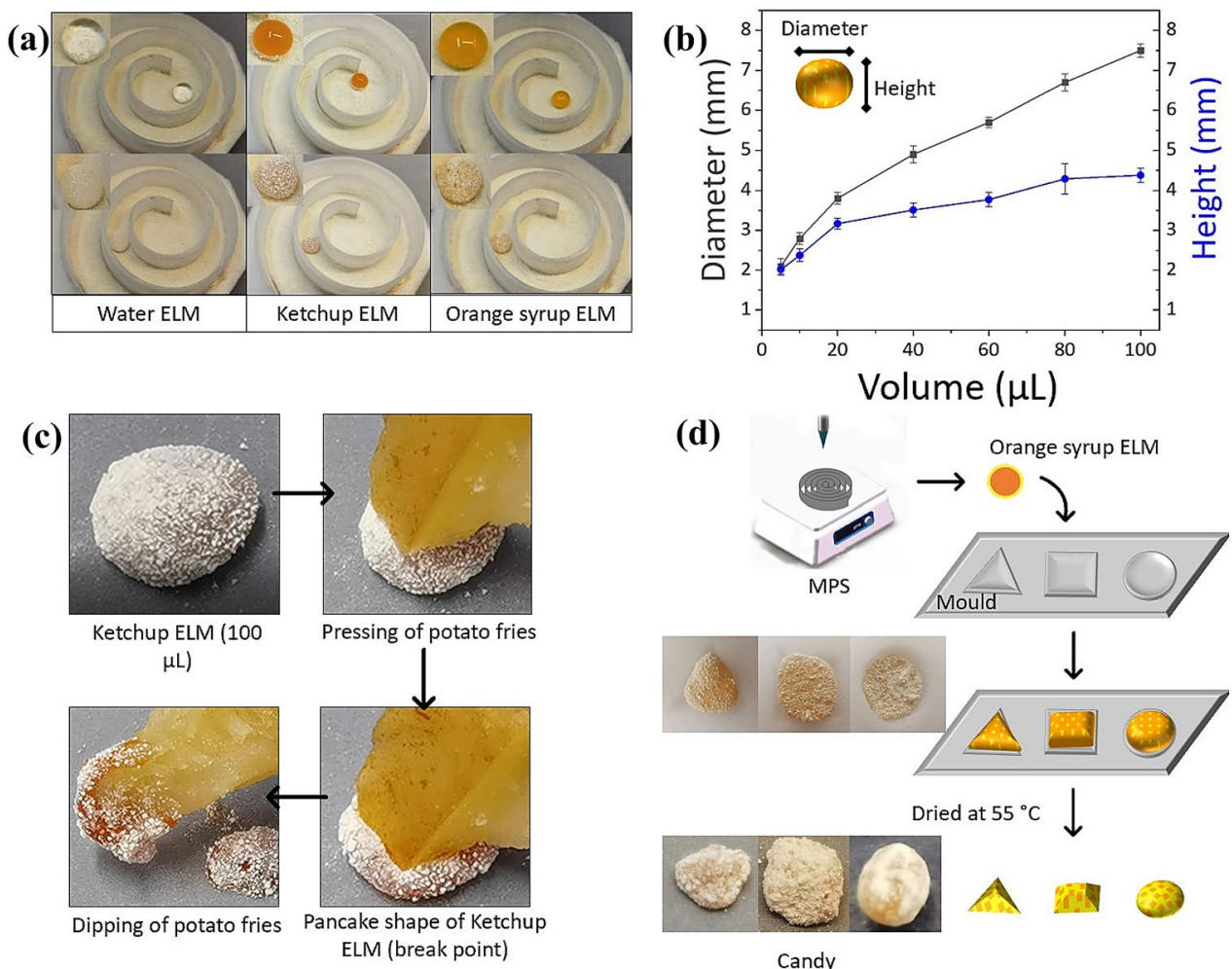

(d)
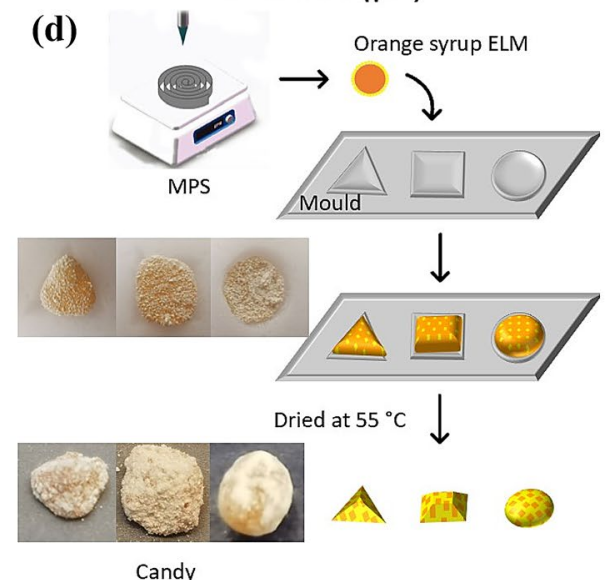

procedures reducing human labour, potential biohazards and flavour loss because the production procedure could be significantly simplified, and enhanced stability of the food against water and water vapour could be achieved [52].

\section{Edible solid marble (ESM)}

Pectin has been widely applied in the pharmaceutical industry as a plasticizer and sustained releasing agent for drugs [38]. The compatibility between pectin and beeswax was also assessed, and pectin could form hydrogen bonds with beeswax and carnauba wax through $-\mathrm{OH}$ groups binding with wax ester groups (Figs. 5a and S1). Consequently, as illustrated in Fig. 5b, the ESM could be fabricated through further drying by applying pectin as a wrapping component of ELM. As observed in Figs. S10-S12, the pectin ESM shrank to a tablet shape when the volume of pectin ELM reached $40 \mu \mathrm{L}$. Figure $5 \mathrm{c}$ illustrates that the pectin ESM can temporarily float on water for approximately $4 \mathrm{~h}$. During this period, the water vapour gradually infiltrates the superhydrophobic coating as the surface bare region, exposing the water-soluble pectin (Figs. 2 and 5d); therefore, breaking the hydrogen bonds between coating and substrate allows direct contact between the pectin and water surface. Furthermore, pectin as a sustained releasing agent could release its content for a further 6-h period, and the wax powder remained floating at the water-air interface due to its superhydrophobicity and superareophilicity. This phenomenon indicates the sustained releasing potential of drugs formulated into ESM.

Water and water vapour accelerate the degradation of solid pectin [53]; thus, the barrier property against water and water vapour is essential in ESM. The heating process of wax has been studied for coating stability and barrier property enhancement [33]. Further heating of mixed wax with components having different melting points $\left(62-64{ }^{\circ} \mathrm{C}\right.$ for beeswax and $82{ }^{\circ} \mathrm{C}$ for carnauba wax) beyond $55{ }^{\circ} \mathrm{C}$ could allow for the wax powder coating with dense layer and eliminate bare regions (Fig. 5d). The wax powder coating with dense beeswax layer could also maintain its superhydrophobicity and eliminate the infiltration of water and water vapour (Fig. 5e). As shown in Fig. 5e, water droplets can maintain a spherical shape on the ESM, and the bottom of ESM on water showed a silver mirror effect indicating the presence of superhydrophobicity [33]. Moreover, the ESM could float on the water surface for at least 7 days with no apparent swelling and disintegration. This long-term floating ability indicates the existence of dense beeswax layer and barrier property against water and water vapour penetration, as well as the floating ability of heated ESM with application fields such as water transportation, antioxidant and intestinal release of medicine. For example, amidated pectin has been researched for the function of transdermal delivery of chloroquine, which is an approved drug for malaria treatment $[54,55]$. 
Fig. 5 Binding mechanism between wax and pectin, and properties of ESM. a Schematic diagram of hydrogen bond binding between wax particles and dried pectin of ESM. b Images of pectin ELM and dried ESM. c Images of disintegration and sustained release of unheated ESM, and SEM image of ESM tablet. d Optical microscopic images of wax coating surface of ELM and heated ESM. e Top: images of heated pectin $\mathrm{ESM}$ on water and $5 \mu \mathrm{L}$ water droplet on pectin ESM; Bottom: pectin ESM after 7 days of water flotation
Fig. 6 Characterization of ESHM and the simulation and analysis of ESHM formation. a Fabrication process of EHM using CPS. b Simulation and illustration of EHM to ESHM drying process with snapshot images taken at $10 \mathrm{~min}$ intervals (images with $5 \mathrm{~mm}$ scale bar). c Optical images of the ESHM and the on-water image and split cross-sectional SEM images of ESHM (a)
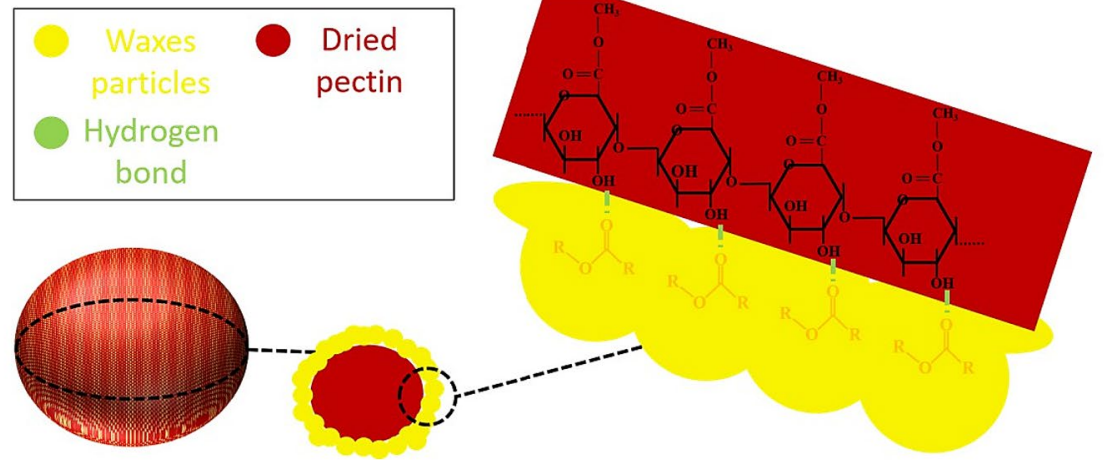

(b)
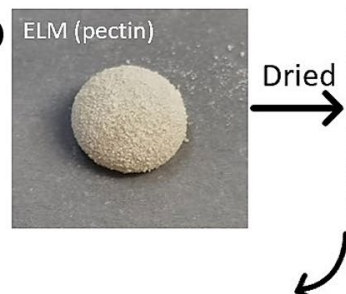

(d)
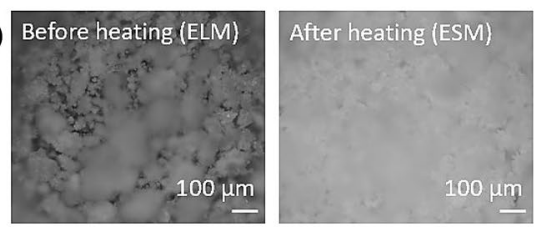

(c)

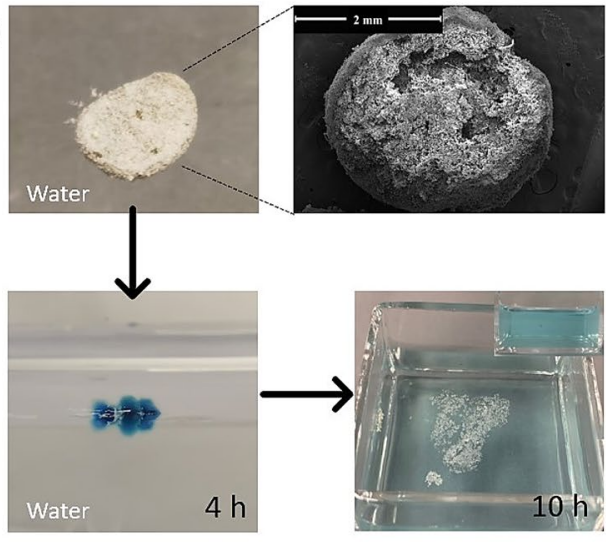

Heated at $55^{\circ} \mathrm{C}$

(e)
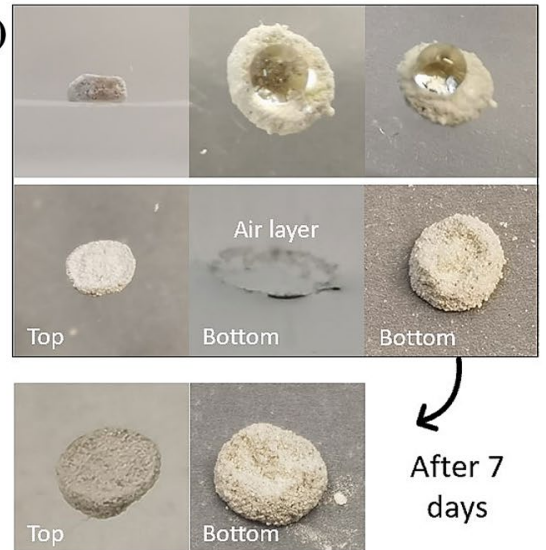

After 7

days
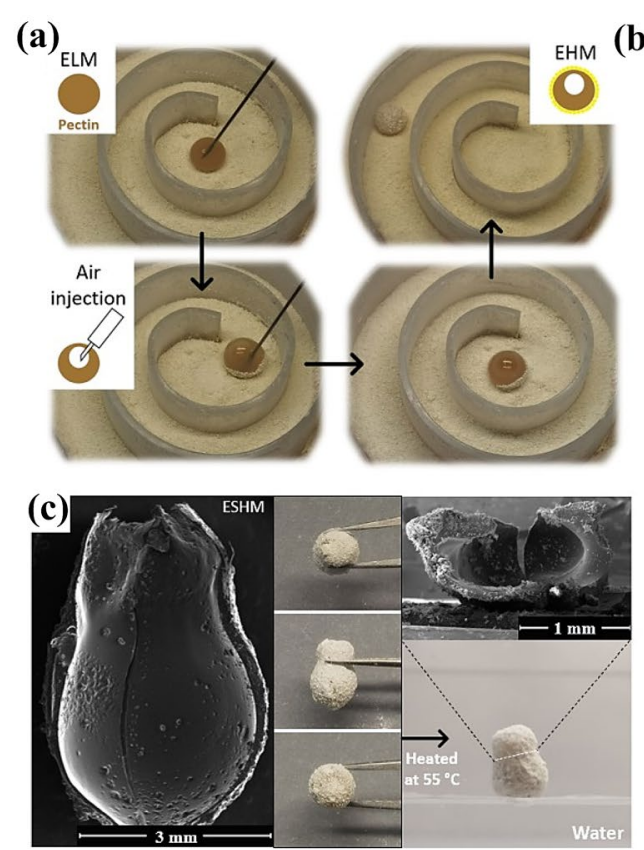
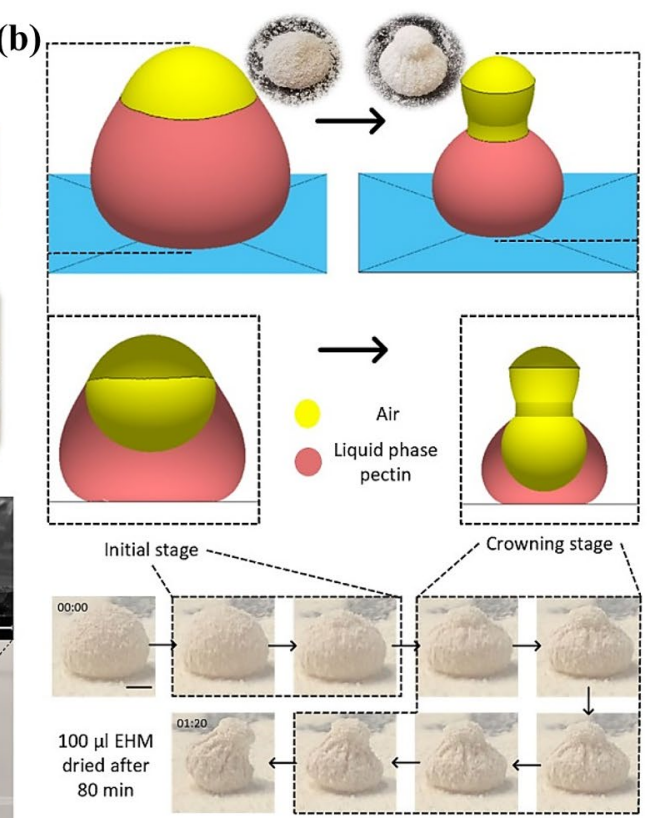


\section{Edible hollow marble (EHM)}

Selected volumes of EHM and ESHM can be produced through air injection at the CPS fabrication stage (Figs. S7 and S8). The intermediate EHM product can be fabricated following CPS production. As illustrated in Fig. 6a, the EHM was fabricated through $100 \mu \mathrm{L}$ pectin ELM with 50 $\mu \mathrm{L}$ air injection. The ESHM was further fabricated by drying at room temperature for around $2 \mathrm{~h}$.

The simulated and actual drying process and shape shift are illustrated in Fig. 6b. The shape of marble resembles an ellipsoid with a convex top, which is attributed to the fact that air is less dense than pectin. To establish the reason of the shape shift initiation and the cause of the final shape, the simulation of marble morphology was conducted via Surface Evolver [56]. Surface tension is considered as the deciding factor, and the surface tension of pectin marble is the same as that of pectin when stretched. The load-bearing ability of a particle raft under the transverse compression of a slender rod was described by Zuo et al. [57]. Accordingly, through surface tension input, the surface tension of pectin is $\gamma=54.88 \mathrm{mN} \cdot \mathrm{m}^{-1}$, and the upper surface tension of the bubble is $109.76 \mathrm{mN} \cdot \mathrm{m}^{-1}$ due to the double air/ pectin surface. The pectin and air density are $1.03 \mathrm{~g} \cdot \mathrm{cm}^{-3}$ and $0.0013 \mathrm{~g} \cdot \mathrm{cm}^{-3}$, respectively. The simulated shapes were created and shown in the top of Fig. 6b, whose crosssectional views are illustrated below. As depicted, there is a bump on the top of the marble at the initial stage, which is consistent with the experimental phenomenon.

First, the top section as the thinnest part of the marble was dried, which became a solid dome. Meanwhile, the pectin tended to shrink with the evaporation of inner water, thus generating tensile stress. To obtain this stress value, pectin droplets with a set volume were placed in the upper dynamometer of a high-sensitivity microelectromechanical balance system (Dataphysics DCAT11, Instruments $\mathrm{GmbH}$, Filderstadt, Germany) and a lower base forming a liquid bridge (Fig. S13). The final force of the liquid bridge was $F_{\mathrm{f}}=1.70 \pm 0.24 \mathrm{mN}$ with the narrowest diameter $D_{\mathrm{f}}=1.22 \pm 0.13 \mathrm{~mm}$. Thus, the tensile stress of liquid pectin can be generated as follows:

$\sigma_{\mathrm{e}}=4 \frac{F_{\mathrm{f}}-\pi \gamma D_{\mathrm{f}}}{\pi D_{\mathrm{f}}^{2}}$.

According to Fig. 6c, the pectin thickness around the middle of the gourd-shaped bubble can be measured as $\delta=0.28 \pm 0.14 \mathrm{~mm}$. Subsequently, the equivalent surface tension of the middle of bubble during evaporation is:

$\gamma_{\mathrm{m}}=2 \gamma+\sigma_{\mathrm{e}} \delta=468.98 \pm 279.54 \mathrm{mN} \cdot \mathrm{m}^{-1}$.

Under the limitation of the top solid dome and the strong equivalent tension in the middle, the ellipsoidal bubble at the initial stage will eventually shrink to a gourd-shaped bubble as shown in lower part of Fig. 6b. Both the shapes of the liquid marble and the bubble are consistent with the experimental results.

Furthermore, as illustrated in Fig. 6c, the images of each angle indicate that the wax powder remained evenly distributed around the gourd ESHM. After heating, the ESHM could maintain flotation on water for at least 7 days without coating loss (Fig. S14). The SEM image of ESHM cross section indicated that the diameter of ESHM neck was around $2.5 \mathrm{~mm}$. The mechanical stability test proved that the ESHM tablet could maintain its gourd shape and surface coating after simulated in-vial transportation (Fig. S15).

The ESHM with vacancy inside can carry oil- or solidphase content when compared with ESM. However, the injection site is necessary for content filling. Fish oil as an important nutritional supplement was selected as content subject. As illustrated in Fig. S16, the heated ESHM maintained its integrity after fish oil injection and acted as waterand airtight capsule for fish oil after hole repair. The ESHM made the capsule enlarged content range of ESM from only pectin-compatible and water-soluble subject to other phases such as solid- or oil-phase subject. Moreover, the ESHM packing process required fewer procedures such as moulding and heat sealing, thus providing lower energy consumption and automated production.

\section{Digestion test of edible solid marble (ESM) and solid hollow marble (ESHM) with temperature-dependent selective releasing ability}

The ESM and ESHM, as illustrated above, could serve as drug or food supplements in the food or pharmaceutical industries. With the addition of mixed wax coating and selective heat-induced vapour barrier property, they could serve as selectively released drugs at the oral or intestine levels. As illustrated in Fig. 7, a series of in vitro digestion tests were conducted to mimic consumption. As the heated ESM and ESHM possessed dense beeswax layers, in contrast to unheated ESM and ESHM, the aqueous digestive solution in Stage I and Stage II could not penetrate the wax coating and dissolve the pectin shell. In digestion Stage III, however, the fat-rich pancreatin mixture could gradually dissolve the wax layer and lead to content release. In this case, as shown in the images of digestion Stage III in Fig. 7, blue-dyed ESM disappeared and red-dyed fish oil was released from the ESHM capsule. The in vitro digestion tests indicated that the tablet-shaped pectin ESM and heated ESM could therefore be applied for pectin-compatible drugs and supplement pills with separate functions: orally released (in vitro digestion, Stage I) or intestine released (in vitro digestion, Stage III) (Table 1) [58-61]. 
Fig. 7 Characterization of flotation/releasing performance of ESM and ESHM in mechanical stability and in vitro digestion tests (Stage I: oral digestion with saliva addition and $\mathrm{pH}_{\text {Stage I }}=6.8$; Stage II: stomach digestion with simulated

gastric fluid and $\mathrm{pH}_{\text {Stage II }}=1.2$; Stage III: intestinal digestion with addition of bile extract and pancreatin mixture and $\mathrm{pH}_{\text {Stage III }}=7$ ). Stage II conditions of headed samples were similar to stage I. On-water/ digestive solution optical images of edible solid marble (ESM) and solid hollow marble (ESHM) after mechanical stability and in vitro digestion tests

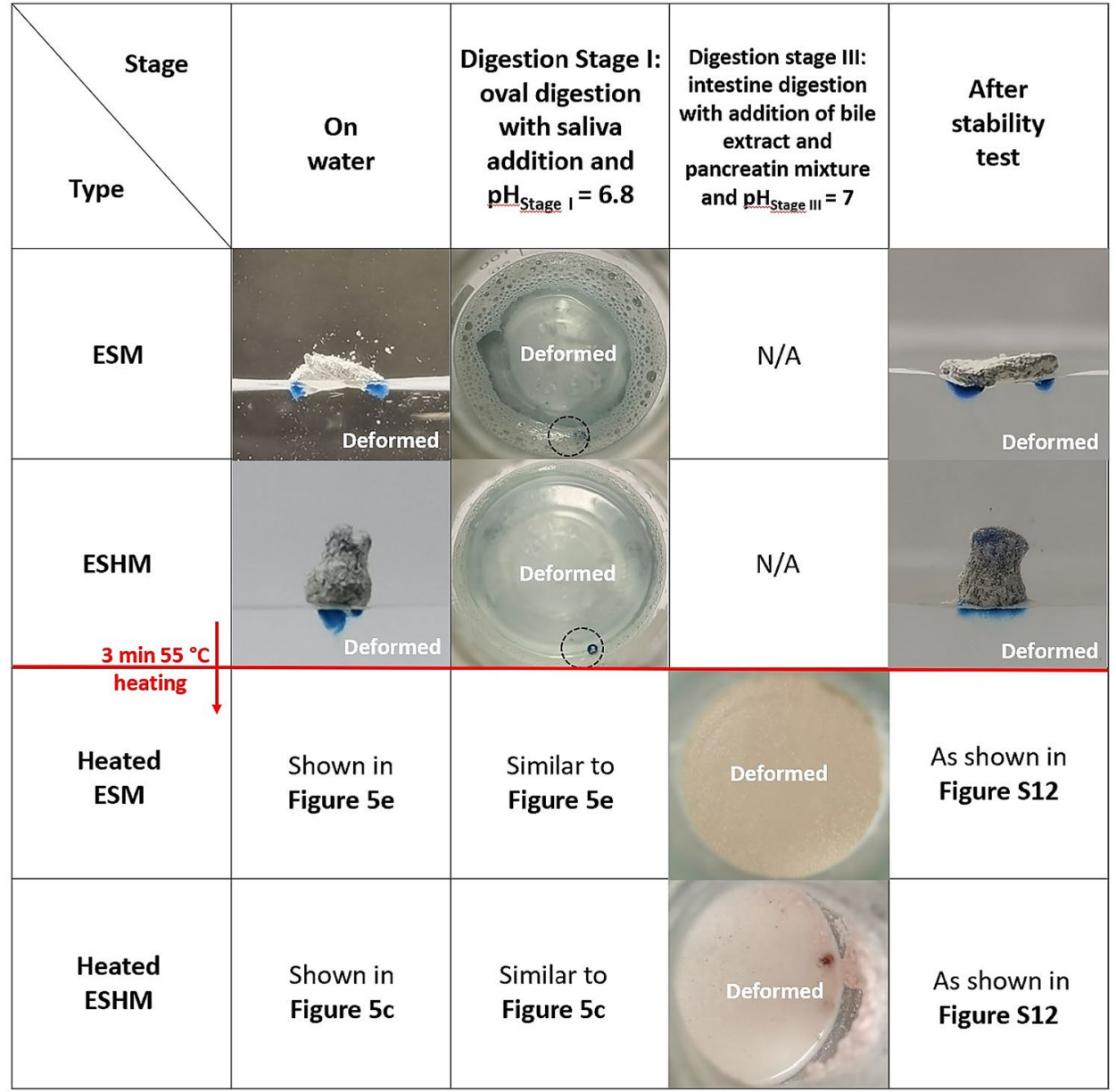

Table 1 Mechanical stability and in vitro digestion tests of edible solid marble (ESM) and solid hollow marble (ESHM)

\begin{tabular}{|c|c|c|c|c|c|c|}
\hline Type & On water & Digestion Stage I & Digestion Stage II & Digestion Stage III & Possible applications & Mechanical stability ${ }^{a}$ \\
\hline ESM & Deforms after $4 h$ & Deforms & N/A & N/A & $\begin{array}{l}\text { Orally released pectin- } \\
\text { compatible drug/sup- } \\
\text { plement or foods }\end{array}$ & Deforms after $4 h$ \\
\hline ESM (heated) & Floats & Floats & Floats & Deforms & $\begin{array}{l}\text { Intestine-released } \\
\text { pectin-compatible } \\
\text { drug/supplement }\end{array}$ & Floats \\
\hline ESHM & Deforms after $4 \mathrm{~h}$ & Deforms & N/A & N/A & $\begin{array}{l}\text { Orally released drug/ } \\
\text { supplement (oil phase) }\end{array}$ & Deforms after $4 \mathrm{~h}$ \\
\hline ESHM (heated) & Floats & Floats & Floats & Deforms & $\begin{array}{l}\text { Intestine-released drug/ } \\
\text { supplement (oil phase) }\end{array}$ & Floats \\
\hline
\end{tabular}

${ }^{a}$ In-vial shaking followed by on-water flotation observation

Deformation indicates the releasing stages of the ESM and ESHM

A mechanical stability test was conducted by in-vial shaking to mimic packaging and transportation of drug and supplement. The mechanical stability test indicated that ESM tablet and ESHM capsule could maintain their sustained release properties after simulation of in-vial transportation (Fig. 7). As shown in Fig. S15, the coating of heated ESM and ESHM remained intact. Therefore, the mechanical stability test indicated that the heated ESM tablet and ESHM capsule could maintain their water and water vapour barrier property after in-vial transportation simulation (Figs. 7 and S15). The performance of heated and unheated ESM and ESHM in the mechanical stability test indicated the 
practicality of drug and capsule applications of ESM and ESHM. Both the coating and capsule of heated and unheated ESM and ESHM remained intact. Therefore, the heated and unheated ESM and ESHM are considered realistic drug or supplement carriers.

\section{Conclusions}

From the aspect of utility of carnauba wax and beeswax [31, $33,35,41]$, this work has demonstrated a new approach for the application of superhydrophobic wax as an independent powder form to substitute wax-in-ethanol emulsion spraying. Moreover, the powdered wax further broadens the current field of research on liquid marbles $[4,8,12,16]$ to that on the food and pharmaceutical industries. In summary, the bioinspired edible liquid marbles and their transition phases, including ESM and ESHM, were mass fabricated through the CPS system. Through the simple heating of wax powder, the heated ESM and ESHM acquired further stability against water and water vapour when compared to previous studies [33], and the ESM and ESHM showed their merits on simple and large-scale production, miniature design and stability against physical impacts. The ELM could pack food liquids and serve as a novel packing and transportation tool for candy beverage making, flavourings and other food additives, leaving no residue and decreasing the biocontamination hazard. The ESM could serve as a tablet for pectin-compactable and water-soluble drug or food supplement with the ability of sustained, selective and temperaturedependent release. In addition, this study first investigated the phase transition of ELM by finite element modelling, providing further reproduction or modification potentials $[7,20]$. The ESHM could function as a selective releasing capsule for oil- or solid-phase drug or food supplement. The CPS production system provided a feasible automated mass production of food packing and carrier for water, oil- and solid-phase drug or supplements. Bioinspired edible liquid marbles therefore have the potential to broaden the field of liquid marble research and be applied in the food or pharmaceutical industries due to their phase transformation characteristics. Moreover, they show research potential in the field of sustained and designated release of medicines or supplements on a clinical level. The phase transition of LM should be explored in further studies, as the solid-phase marble may possess the properties of currently researched LM and hold its integrity. The form of wax powder as a superhydrophobic interfacial surfactant could also be explored for other materials. For instance, it could replace traditional wax-in-ethanol emulsion spraying in the field of anti-adhesion superhydrophobic modification of hydrophilic surfaces, such as glass and plastics.
Supplementary Information The online version contains supplementary material available at https://doi.org/10.1007/s42242-021-00158-z.

Acknowledgements This work was financially supported by the National Natural Science Foundation of China (No. 51735013).

Author contributions DHW designed the project, contributed to the experiment conduction and manuscript writing. SPL had contributed to finite element modelling and analysis. BW had contributed to the manuscript processing and refining. ZGG and WML had contributed to the idea for the manuscript and revised it with the support of finance. All authors commented on the paper.

\section{Declarations}

Conflict of interest The authors declare that they have no known competing financial interests or personal relationships that could have appeared to influence the work reported in this paper.

Ethical approval This article does not contain any studies with human or animal subjects performed by any of the authors.

\section{References}

1. Pike N, Richard D, Foster W et al (2002) How aphids lose their marbles. Proc Biol Sci 269(1497):1211-1215. https://doi.org/10. 1098/rspb.2002.1999

2. Kasahara M, Akimoto S, Hariyama T et al (2019) Liquid marbles in nature: craft of aphids for survival. Langmuir 35(18):61696178. https://doi.org/10.1021/acs.langmuir.9b00771

3. Tian J, Arbatan T, Li X et al (2010) Liquid marble for gas sensing. Chem Commun 46(26):4734-4736. https://doi.org/10.1039/ C001317J

4. Yukioka S, Fujiwara J, Okada $\mathrm{M}$ et al (2020) $\mathrm{CO}_{2}$-gas-responsive liquid marble. Langmuir 36(25):6971-6976. https://doi.org/10. 1021/acs.langmuir.9b03074

5. Sato E, Yuri M, Fujii S et al (2016) Liquid marble containing degradable polyperoxides for adhesion force-changeable pressuresensitive adhesives. RSC Adv 6(61):56475-56481. https://doi.org/ 10.1039/C6RA10677C

6. Lin KJ, Chen RY, Zhang LY et al (2019) Transparent bioreactors based on nanoparticle-coated liquid marbles for in situ observation of suspending embryonic body formation and differentiation. ACS Appl Mater Interfaces 11(9):8789-8796. https://doi.org/10. 1021/acsami.8b20169

7. Zhao Y, Xu ZG, Niu HT et al (2015) Magnetic liquid marbles: toward "lab in a droplet." Adv Funct Mater 25(3):437-444. https:// doi.org/10.1002/adfm.201403051

8. Wang B, Liu Y, Zhang YB et al (2015) Bioinspired superhydrophobic $\mathrm{Fe}_{3} \mathrm{O}_{4} @$ polydopamine@ Ag hybrid nanoparticles for liquid marble and oil spill. Adv Mater Interfaces 2(13):1500234. https:// doi.org/10.1002/admi.201500234

9. Dandan M, Erbil HY (2009) Evaporation rate of graphite liquid marbles: comparison with water droplets. Langmuir 25(14):83628367. https://doi.org/10.1021/la900729d

10. Cengiz U, Erbil HY (2013) The lifetime of floating liquid marbles: the influence of particle size and effective surface tension. Soft Matter 9(37):8980-8991. https://doi.org/10.1039/c3sm51304a

11. Sreejith KR, Ooi CH, Dao DV et al (2018) Evaporation dynamics of liquid marbles at elevated temperatures. RSC Adv 8(28):15436-15443. https://doi.org/10.1039/C8RA02265H 
12. Wang B, Chan KF, Ji F et al (2019) On-demand coalescence and splitting of liquid marbles and their bioapplications. Adv Sci 6(10):1802033. https://doi.org/10.1002/advs.201802033

13. Fujii S, Yusa S, Nakamura Y et al (2016) DD stimuli-responsive liquid marbles: controlling structure, shape, stability, and motion. Adv Funct Mater 26(40):7206-7223. https://doi.org/10.1002/ adfm.201603223

14. Kim SH, Lee SY, Yang SM (2010) Janus microspheres for a highly flexible and impregnable water-repelling interface. Angew Chem Int Edit 49(14):2535-2538. https://doi.org/10.1002/ange.20100 0770

15. Zhao Y, Fang J, Wang HX et al (2010) Magnetic liquid marbles: manipulation of liquid droplets using highly hydrophobic $\mathrm{Fe}_{3} \mathrm{O}_{4}$ nanoparticles. Adv Mater 22(6):707-710. https://doi.org/10.1002/ adma.200902512

16. Khaw MK, Ooi CH, Mohd-Yasin F et al (2017) Dynamic behaviour of a magnetically actuated floating liquid marble. Microfluid Nanofluid 21(6):110. https://doi.org/10.1007/s10404-017-1945-0

17. Wang B, Chan KF, Yuan K et al (2021) Endoscopy-assisted magnetic navigation of biohybrid soft microrobots with rapid endoluminal delivery and imaging. Sci Robot 6(52):abd2813. https:// doi.org/10.1126/scirobotics.abd2813

18. Paven M, Mayama H, Sekido T et al (2016) Light-driven delivery and release of materials using liquid marbles. Adv Funct Mater 26(19):3199-3206. https://doi.org/10.1002/adfm.201600034

19. Kavokine N, Anyfantakis M, Morel M et al (2016) Light-driven transport of a liquid marble with and against surface flows. Angew Chem Int Edit 55(37):11183-11187. https://doi.org/10.1002/anie. 201603639

20. Tenjimbayashi M, Samitsu S, Naito M (2019) Simultaneous detection and repair of wetting defects in superhydrophobic coatings via Cassie-Wenzel transitions of liquid marbles. Adv Funct Mater 29(26):1900688. https://doi.org/10.1002/adfm.201900688

21. Ellis DA, Mabury SA, Martin JW et al (2001) Thermolysis of fluoropolymers as a potential source of halogenated organic acids in the environment. Nature 412(6844):321-324. https://doi.org/10. $1038 / 35085548$

22. Suja F, Pramanik BK, Zain SM (2009) Contamination, bioaccumulation and toxic effects of perfluorinated chemicals (PFCs) in the water environment: a review paper. Water Sci Technol 60(6):1533-1544. https://doi.org/10.2166/wst.2009.504

23. Johansson N, Fredriksson A, Eriksson P (2008) Neonatal exposure to perfluorooctane sulfonate (PFOS) and perfluorooctanoic acid (PFOA) causes neurobehavioural defects in adult mice. Neurotoxicology 29(1):160-169. https://doi.org/10.1016/j.neuro.2007. 10.008

24. Cliver DO (1997) Virus transmission via food. Food Technol Chic 51(4):71-78

25. Hobbs JE (2020) Food supply chains during the COVID-19 pandemic. Can J Agr Econ 68(2):171-176. https://doi.org/10.1111/ cjag. 12237

26. Galanakis CM (2020) The food systems in the era of the coronavirus (COVID-19) pandemic crisis. Foods 9(4):523. https://doi. org/10.3390/foods 9040523

27. Seleiman MF, Selim S, Alhammad BA et al (2020) Will novel coronavirus (Covid-19) pandemic impact agriculture, food security and animal sectors? Biosci J 36(4):1315-1326. https://doi.org/ 10.14393/BJ-v36n4a2020-54560

28. Cromeans TL (1997) Understanding and preventing virus transmission via foods. Food Technol Chic 51(4):20

29. Janjarasskul T, Krochta JM (2010) Edible packaging materials. Annu Rev Food Sci Technol 1:415-448. https://doi.org/10.1146/ annurev.food.080708.100836

30. Kawamura Y, Mayama H, Nonomura Y (2012) Edible liquid marbles and capsules covered with lipid crystals. J Oleo Sci 61(9):477-482. https://doi.org/10.5650/jos.61.477
31. Wang W, Lockwood K, Boyd LM et al (2016) Superhydrophobic coatings with edible materials. Acs Appl Mater Interfaces 8(29):18664-18668. https://doi.org/10.1021/acsami.6b06958

32. Zhang Y, Bi J, Wang S et al (2019) Functional food packaging for reducing residual liquid food: thermo-resistant edible super-hydrophobic coating from coffee and beeswax. J Colloid Interface Sci 533:742-749. https://doi.org/10.1016/j.jcis.2018. 09.011

33. Wang D, Huang J, Guo Z (2020) Tomato-lotus inspired edible superhydrophobic artificial lotus leaf. Chem Eng J 400:125883. https://doi.org/10.1016/j.cej.2020.125883

34. Liu BY, Xue CH, An QF et al (2019) Fabrication of superhydrophobic coatings with edible materials for super-repelling non-Newtonian liquid foods. Chem Eng J 371:833-841. https:// doi.org/10.1016/j.cej.2019.03.222

35. Li Y, Bi J, Wang S et al (2018) Bio-inspired edible superhydrophobic interface for reducing residual liquid food. J Agric Food Chem 66(9):2143-2150. https://doi.org/10.1021/acs.jafc.7b059 15

36. Zhao X, Hu T, Zhang J (2018) Superhydrophobic coatings with high repellency to daily consumed liquid foods based on food grade waxes. J Colloid Interface Sci 515:255-263. https://doi.org/ 10.1016/j.jcis.2018.01.034

37. Yang C, Zhu Y, Wang Y et al (2019) Preparation of edible superhydrophobic $\mathrm{Fe}$ foil with excellent stability and durability and its applications in food containers with little residue. New J Chem 43(7):2908-2919. https://doi.org/10.1039/c8nj05967e

38. Rowe RC, Sheskey PJ, Quinn ME (2009) Handbook of Pharmaceutical Excipients, 6th edn. Pharmaceutical Press, American Pharmacists Association, London

39. Sinha VR, Mittal BR, Kumria R (2005) In vivo evaluation of time and site of disintegration of polysaccharide tablet prepared for colon-specific drug delivery. Int J Pharm 289(1-2):79-85. https:// doi.org/10.1016/j.ijpharm.2004.10.019

40. Sweetman SC (2005) Dose adjustment in renal impairment: response from martindale: the complete drug reference. Br Med J 331(7511):292-293. https://doi.org/10.1136/bmj.331.7511.292-a

41. Wang D, Guo Z, Liu W (2019) Bioinspired edible lubricantinfused surface with liquid residue reduction properties. Research 2019:1649427. https://doi.org/10.34133/2019/1649427

42. Gawlik-Dziki U, Dziki D, Baraniak B et al (2009) The effect of simulated digestion in vitro on bioactivity of wheat bread with Tartary buckwheat flavones addition. LWT Food Sci Technol 42(1):137-143. https://doi.org/10.1016/j.lwt.2008.06.009

43. Bhushan B, Jung YC, Koch K (2009) Micro-, nano- and hierarchical structures for superhydrophobicity, self-cleaning and low adhesion. Philos Trans Royal Soc A 367(1894):1631-1672. https://doi.org/10.1098/rsta.2009.0014

44. Matulis D, Bloomfield VA (2002) Determination of hydrophobic interactions between long chain aliphatic hydrocarbons by titration calorimetry. Biophys J 82(1):335a-336a

45. McEleney P, Walker GM, Larmour IA et al (2009) Liquid marble formation using hydrophobic powders. Chem Eng J 147(23):373-382. https://doi.org/10.1016/j.cej.2008.11.026

46. Kubo W, Miyazaki S, Dairaku M et al (2004) Oral sustained delivery of ambroxol from in situ-gelling pectin formulations. Int $\mathrm{J}$ Pharm 271(1-2):233-240. https://doi.org/10.1016/j.ijpharm.2003. 11.027

47. Murata Y, Miyashita M, Kofuji K et al (2004) Drug release properties of a gel bead prepared with pectin and hydrolysate. J Control Release 95(1):61-66. https://doi.org/10.1016/j.jconrel.2003. 10.026

48. Kulinich SA, Farzaneh M (2009) Effect of contact angle hysteresis on water droplet evaporation from super-hydrophobic surfaces. Appl Surf Sci 255(7):4056-4060. https://doi.org/10.1016/j. apsusc.2008.10.109 
49. Planchette C, Biance AL, Pitois O et al (2013) Coalescence of armored interface under impact. Phys Fluids 25(4):042104. https://doi.org/10.1063/1.4801320

50. Muller MG, Lindner JA, Briesen $\mathrm{H}$ et al (2018) On the properties and application of beeswax, carnauba wax and palm fat mixtures for hot melt coating in fluidized beds. Adv Powder Technol 29(3):781-788. https://doi.org/10.1016/j.apt.2017.12.020

51. Singh S, Singh B, Alam T (2019) Evaluation of shelf-life, antioxidant activity and nutritional quality attributes in carnauba wax coated eggplant genotypes. J Food Sci Technol 56(11):48264833. https://doi.org/10.1007/s13197-019-03944-x

52. Fabri I, Deak T, Farkas J (1988) Microbiological control of foodprocessing on the basis of the hazard analysis, critical control points (haccp) system. Acta Microbiol Hung 35(2):152

53. Salazar ASS, Cavazos PAS, Paz HM et al (2019) External factors and nanoparticles effect on water vapor permeability of pectinbased films. J Food Eng 245:73-79. https://doi.org/10.1016/j.jfood eng.2018.09.002

54. Musabayane CT, Munjeri O, Matavire TP (2003) Transdermal delivery of chloroquine by amidated pectin hydrogel matrix patch in the rat. Ren Fail 25(4):525-534. https://doi.org/10.1081/JDI120022543

55. Hu TY, Frieman M, Wolfram J (2020) Insights from nanomedicine into chloroquine efficacy against COVID-19. Nat Nanotechnol 15(4):247-249. https://doi.org/10.1038/s41565-020-0674-9
56. Brakke KA (1996) The surface evolver and the stability of liquid surfaces. Philos Trans Royal Soc A 354(1715):2143-2157. https:// doi.org/10.1098/rsta.1996.0095

57. Zuo PC, Liu JL, Li SP (2017) The load-bearing ability of a particle raft under the transverse compression of a slender rod. Soft Matter 13(12):2315-2321. https://doi.org/10.1039/c6sm02752k

58. Sriamornsak P, Nunthanid J, Wanchana S et al (2003) Composite film-coated tablets intended for colon-specific delivery of 5-aminosalicylic acid: using deesterified pectin. Pharm Dev Technol 8(3):311-318. https://doi.org/10.1081/PDT-120022159

59. Liu LS, Fishman ML, Kost J et al (2003) Pectin-based systems for colon-specific drug delivery via oral route. Biomaterials 24(19):3333-3343. https://doi.org/10.1016/S0142-9612(03) 00213-8

60. Tho I, Sande SA, Kleinebudde P (2003) Disintegrating pellets from a water-insoluble pectin derivative produced by extrusion/ spheronisation. Eur J Pharm Biopharm 56(3):371-380. https:// doi.org/10.1016/S0939-6411(03)00071-7

61. Chourasia MK, Jain SK (2003) Pharmaceutical approaches to colon targeted drug delivery systems. J Pharm Pharm Sci $6(1): 33-66$ 